\title{
Effects of Cellphone Radiation on Sperm Quality and the Expression of StAR in the Testis of C57BL/6 Mice
}

\author{
Zhi-Jun Zang1, Yong Gao², Shi-Zong Huang1, Su-Yun Ji³, Mei-Hua Jiang4, Jiong Shu ${ }^{5 *}$ \\ ${ }^{1}$ Department of Infertility and Sexual Medicine, The Third Affiliated Hospital, Sun Yat-sen University, Guangzhou, China \\ ${ }^{2}$ Reproductive Medicine Centre and Guangdong Provincial Key Laboratory of Reproductive Medicine, The First Affiliated Hos- \\ pital, Sun Yat-sen University, Guangzhou, China \\ ${ }^{3}$ Department of Dermatology, Guangdong Provincial Dermatology Hospital, Guangzhou, China \\ ${ }^{4}$ Department of Anatomy, Zhongshan School of Medicine, Sun Yat-sen University, Guangzhou, China \\ ${ }^{5}$ Department of Endocrinology, The Third Affiliated Hospital, Sun Yat-sen University, Guangzhou, China \\ Email: *shujiongzssy@163.com
}

How to cite this paper: Zang, Z.-J., Gao, Y., Huang, S.-Z., Ji, S.-Y., Jiang, M.-H. and Shu, J. (2017) Effects of Cellphone Radiation on Sperm Quality and the Expression of StAR in the Testis of C57BL/6 Mice. Occupational Diseases and Environmental Medicine, 5, 79-87.

https://doi.org/10.4236/odem.2017.54008

Received: September 18, 2017

Accepted: October 29, 2017

Published: November 1, 2017

Copyright $\odot 2017$ by authors and Scientific Research Publishing Inc. This work is licensed under the Creative Commons Attribution International License (CC BY 4.0).

http://creativecommons.org/licenses/by/4.0/

\begin{abstract}
Background: It has been reported that cellphone radiation (CR) is related to higher risk of many health problems, but whether CR can impair the expression of rate-limiting enzymes of testosterone synthesis has seldom been studied. Objective: To evaluate the effects of CR on the expression of steroidogenic acute regulatory protein (StAR) in the testes tissue and the sperm quality of adult male mice. Methods: Forty 3-month-old male mice, $22-26 \mathrm{~g}$, were randomly assigned into four equal groups ( $\mathrm{n}=10$ per group): the control group and three $\mathrm{CR}$ exposure groups including 8-hour group, 16-hour group and 24-hour group. Each mouse received different dosages of CR exposure for seven consecutive weeks. Semen in the epididymis, intratesticular testosterone (ITT) concentrations, and the expression of StAR were measured at the end of experiment. Results: The sperm number and motility, and the ITT concentrations in 24-h group were significant lower than those in the control group ( $\mathrm{P}$ $<0.05, \mathrm{P}<0.01$, and $\mathrm{P}<0.05$ respectively). No obvious changes were seen in 8-hour group and 16-hour group compared with the control (both $\mathrm{P}>0.05$ ). Similarly, only the expression of StAR in the 24-h group was significantly decreased after the exposure of $\mathrm{CR}(\mathrm{P}<0.05)$. Both the 8-h group and the 16-h group did not change much in the expression of StAR compared with the control group ( $\mathrm{P}>0.05)$. Conclusions: High dose exposure of $\mathrm{CR}$ can reduce the expression of StAR and ITT concentration, and then suppress the serum quality.
\end{abstract}




\section{Keywords}

Cellphone Radiation, Sperm Quality, Testosterone, StAR

\section{Introduction}

Nowadays most men own cellphones, and this phenomenon arouse the concern over the potential effects of cellphone radiation (CR) exposure on human health, including human fertility. In fact, it has been proved that cellphone can emit electromagnetic radiation at a frequency of between 800 and $2200 \mathrm{MHz}$ and can be absorbed by the body [1]. Many potential adverse effects of CR exposure have been reported, including high resting blood pressure [2], headaches [3] and disturbances to electroencephalographic activity during sleep [4]. Some researches suggested that CR could reduce sperm quality [5] [6], but the mechanism has not been completely clarified. Our previous studies showed that $\mathrm{CR}$ can decline the testosterone and LH levels in mice and inhibit their sexual behaviors [7]. Leydig cells are the main source of testosterone in vivo, which can be modulated by the hypothalamic-pituitary-gonadal (HPG) axis. Normally, intratesticular testosterone (ITT) concentrations are roughly 50 - 100 times serum levels and are critical for spermatogenesis [8]. Suppression of ITT production could compromise spermatogenesis and even result in azoospermia [8]. The aim of this study was to assess whether CR could affect the level of ITT and decrease the sperm quality of mice. Moreover, we tested the expression of one rate-limiting enzymes of testosterone synthesis, steroidogenic acute regulatory protein (StAR), to elucidate the mechanism of CR action in this process.

\section{Materials and Methods}

\subsection{Animals}

Forty 3-month-old male C57BL/6 mice (weighted $22 \mathrm{~g} \pm 3 \mathrm{~g}$ ) were purchased from the Animal Center of Sun Yat-sen University (Guangzhou, China) and were kept on a $12 \mathrm{~h}$-day/12 h-night schedule (lights on from 19:00 to 07:00 h) at constant temperature $\left(22 \pm 1^{\circ} \mathrm{C}\right)$ and humidity $(60 \%)$. The present experiment was supervised by the Sun Yat-sen University Institutional Animal Care and Use Committee.

All of the male mice were randomly arranged into four experimental groups (10 mice per group): one control group and three CR-exposure groups receiving different dosage of CR ( 8 hours, 16 hours or 24 hours per day) following to the method mentioned in former articles [9] [10]. The radiofrequency of these cellphones is $900 \mathrm{MHz}$ of global system for cell (GSM). Each experimental group received different dose of GSM exposure for seven consecutive weeks. Twenty-four hours after the last feeding, the mice were sacrificed and the organs were harvested. 


\subsection{Semen Analysis}

After the experiment, the excised epididymides were cut into small pieces and placed in $1 \mathrm{ml} \mathrm{F} 12$ media containing $0.1 \%$ bovine serum albumin prewarmed to $34^{\circ} \mathrm{C}$ and incubated for $15 \mathrm{~min}$ to facilitate sperm transmigration from the epididymis. The number of sperm was counted using a hemocytometer. Each specimen was counted twice and averaged. Fresh sperm was loaded on a prewarmed glass slide to examine sperm motility by microscopy. Four randomly chosen fields of each sample were selected to assess sperm motility. The percentage of sperm with forward and progressive activity was counted to assay sperm motility.

\subsection{Intratesticular Testosterone Analysis}

The ITT was measured according to the following method: The right testis of each mouse was harvested and decapsulated. Two pieces weighted $50 \mathrm{mg}$ each were picked out and were placed separately into two $1.5 \mathrm{ml}$ microfuge tubes containing $1.0 \mathrm{ml}$ Medium 199 (Corning Cellgro, VA, USA). Then, the testis tissue was incubated for $2 \mathrm{~h}$ at $34^{\circ} \mathrm{C}$, and then centrifuged for $5 \mathrm{~min}$ at a speed of $10,000 \times \mathrm{g}$. The supernatant was collected and frozen at $-80^{\circ} \mathrm{C}$ for future testosterone assay. Testosterone assay was conducted using a commercially available enzyme-linked immunosorbent assay kit (R \& D Systems, MN, USA) following the manufacturer's instructions.

\subsection{Immunofluorescence Staining}

Testis tissue fixed with $4 \%$ paraformaldehyde werecryo-embedded in an optimal cutting temperature medium (Sakura Finetek, Torrance, CA, USA) and were sectioned at a thickness of $4 \mu \mathrm{m}$. The sections were blocked by incubation in 5\% normal serum and $0.1 \%$ Triton X-100 (Hyclone, Logan, Utah, USA) in PBS for 1 $\mathrm{h}$ at room temperature, and then incubated with primary antibody (Mouse polyclonal to StAR (D-2), 1:200, Santa Cruz, catalog number: sc-166821) overnight at $4^{\circ} \mathrm{C}$. The sections were incubated with secondary antibody (Goat anti-mouse IgGAlexa594, 1:1000 Invitrogen, Carlsbad, CA, USA, catalog number: A11032) for $30 \mathrm{~min}$ at room temperature followed by DAPI (blue, 1:1 Millipore, Darmstadt, Germany, catalog number: S7113) staining of nuclei next day. PBS replaced the primary antibody as a negative control. All the images were captured using an LSM710 confocal microscope (Zeiss, Jena, Germany) and were analyzed using the Image J software (University Health Network, USA).

\subsection{Quantitative Real-Time PCR}

Total RNA from the testes of these mice was extracted using an RNeasy mini kit (Qiagen, Tokyo, Japan) following the manufacturer's instruction. Reverse transcription reactions were performed by utilizing murine leukemia virus reverse transcriptase and oligo-dT primers (Fermentas, Lithuania). Real-time PCR was conducted using the ThunderbirdSYBR qPCR Mix (Toyobo, Osaka, Japan) ac- 
cording to the protocol of the manufacturer's. $\beta$-actin mRNA was selected as an internal control. A Light Cycler 480 Detection System (Roche, Basel, Switzerland) was chosen to detect signals. The primer sequences are depicted as follows: 5-GGGCATACTCAACAACCAG-3 (upper primer) and 5-ACCTCCAGTCGGAACACC-3 (lower primer) for StAR, and 5-TCGTGCGTGACATTAACGAG-3 (upper primer) and 5-ATTGCCTATCGTGATGACCT-3 (lower primer) for $\beta$-actin.

\subsection{Statistical Analysis}

Results were presented as mean \pm s.e.m. One-way ANOVA was utilized to assess the significance of differences in each group. In all cases, $\mathrm{P}<0.05$ was considered to be statistically significant. The statistical data were analyzed by using SPSS, version 21.0 (SPSS Inc., Chicago, IL, USA). All analytic results were performed using the GraphPad Software package (GraphPad Software, La Jolla, CA, USA).

\section{Results}

\subsection{Results of Semen Analysis}

To determine whether sperm number and motility changed after the exposure of $\mathrm{CR}$, epididymal sperm from the mice were analyzed. Results indicated that there was a significant decrease in sperm number and motility in the 24-h group compared with the control group (sperm number: $[9.34 \pm 3.87] \times 10^{6}$ cells $/ \mathrm{ml}$ vs. [14.19 \pm 4.85$] \times 10^{6}$ cells $/ \mathrm{ml}, \mathrm{P}<0.05$; sperm motility: [27.73 \pm 7.45$] \%$ vs. 41.21 $\pm 10.00 \%, \mathrm{P}<0.01$ ) (Figure 1 ). There were no obvious difference between the other two experimental groups and the control group.

\subsection{Outcomes of ITT}

As shown in Figure 2, the ITT of the 24-hour group was significantly decreased compared with that of the control group $([76.20 \pm 9.17] \mathrm{ng} / \mathrm{g}$ vs. [85.75 \pm 8.01$]$ $\mathrm{ng} / \mathrm{g}, \mathrm{P}<0.05)$. No significant differences in ITT were seen between the control group and the other two experimental groups $(\mathrm{P}>0.05)$.

\subsection{Immunofluorescence Staining Results}

The effects of CR at different exposure time on the expression of StAR are shown in Figure 3. The number of StAR-positive Leydig cells in 24-h group was obviously smaller than that of the control group $(2.90 \pm 1.79$ vs. $4.80 \pm 2.04, \mathrm{P}<$ 0.05). Similarly, exposure to $C R$ at the duration of 8 hours and 16 hours also did not affect the expression of StAR in Leydig cells (both $\mathrm{P}>0.05$ ).

\subsection{Quantitative Real-Time PCR Results}

The effect of CR exposure on the expression levels of StAR genes was determined by quantitative real-time PCR (Figure 4). Only the mRNA level of StAR in 24-h group was markedly decreased compared with the control group $(0.92 \pm 0.09$ vs. 


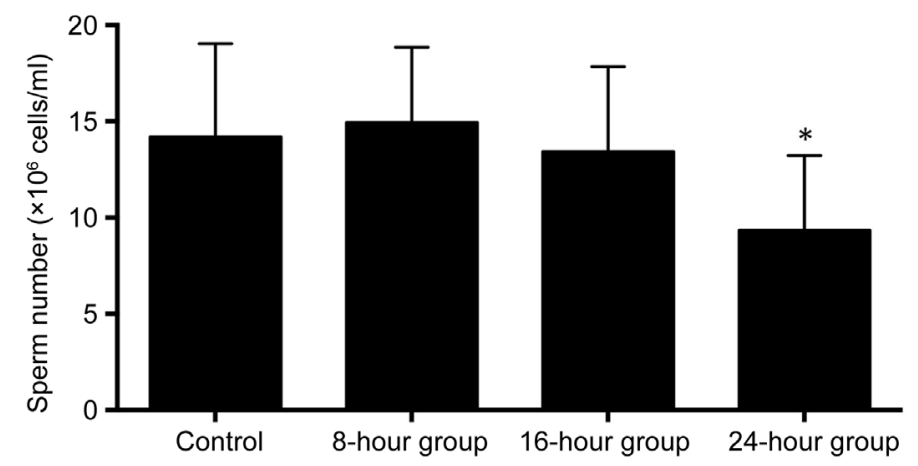

(a)

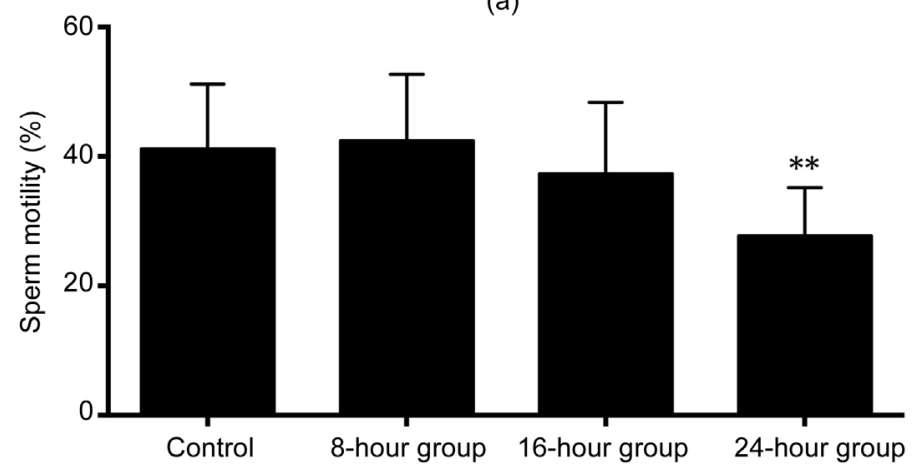

(b)

Figure 1. Alterations in sperm number and sperm motility after exposure to cellphone radiation (CR). (a) Changes in sperm number; (b)Changes in sperm motility. Data are shown as mean \pm standard error of the mean; ${ }^{\star} \mathrm{P}<0.05,{ }^{*} \mathrm{P}<0.01 ; \mathrm{n}=10$ /group.

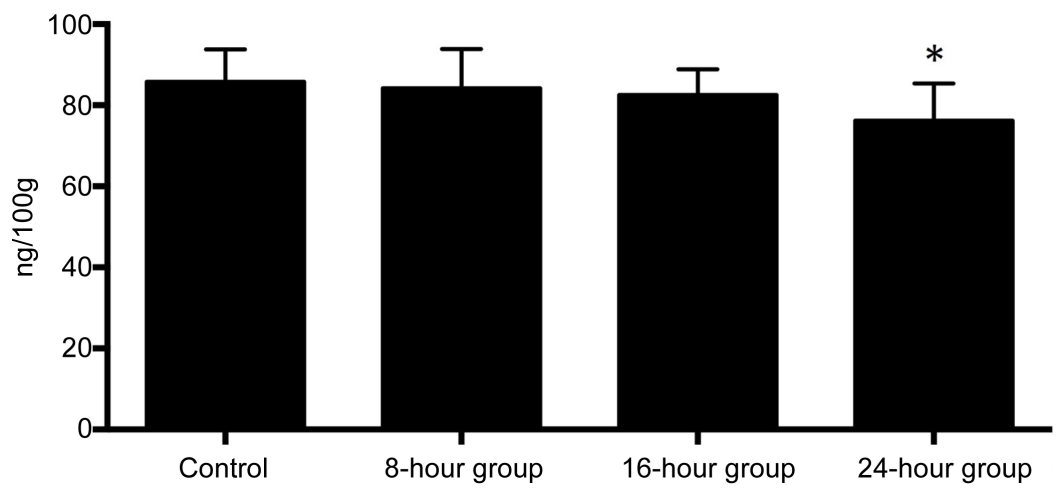

Figure 2. Effects of $\mathrm{CR}$ on the levels of intratesticular testosterone. All values are mean \pm standard error of the mean; ${ }^{*} \mathrm{P}<0.05$ compared with controlgroup; $\mathrm{n}=10$ /group.

$1, \mathrm{P}<0.05)$. No statistical differences were seen between the other two experimental groups and the control group (both $\mathrm{P}>0.05$ ).

\section{Discussion}

We have previously proven that exposure to CR could decrease the serum testosterone concentration and inhibit sexual behaviors of adult male mice. This study further verifies that CR exposure can also decrease the ITT and sperm quality and demonstrates for the first time that CR exposure can reduce the ex- 


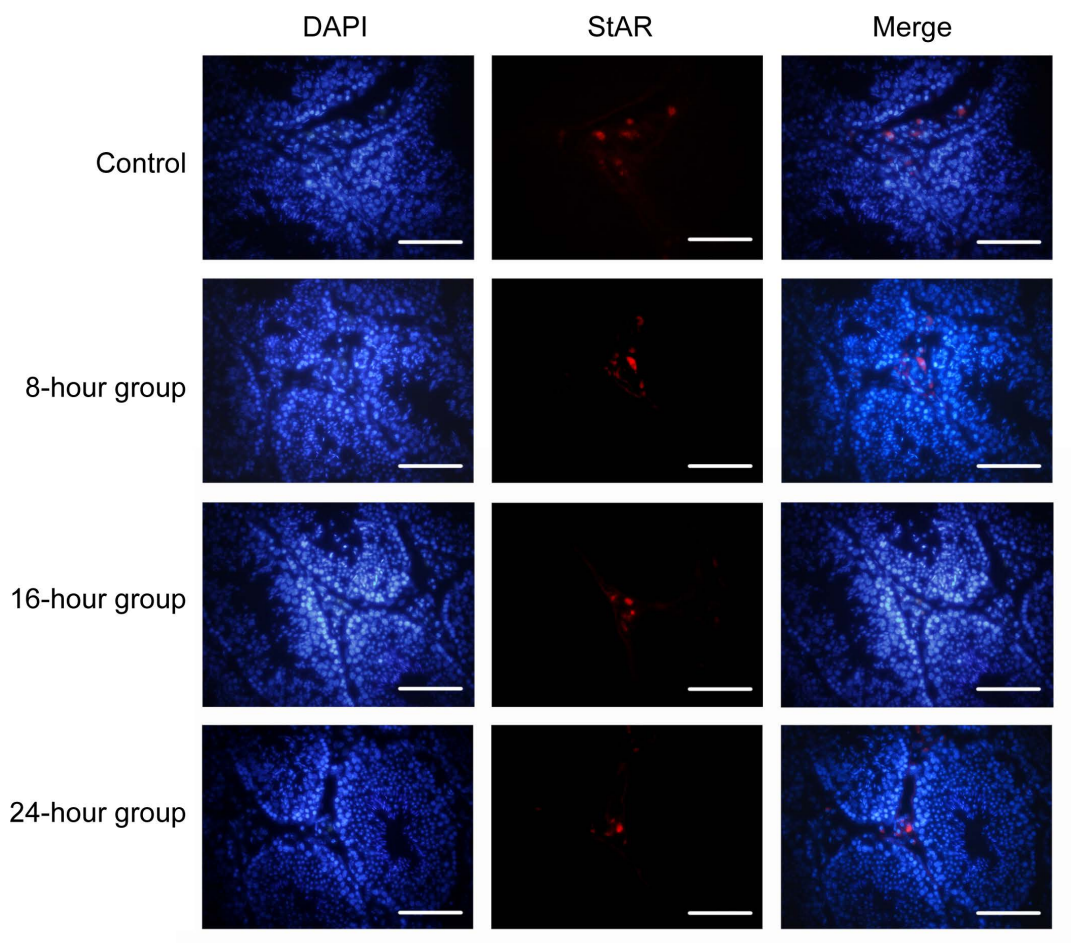

(a)

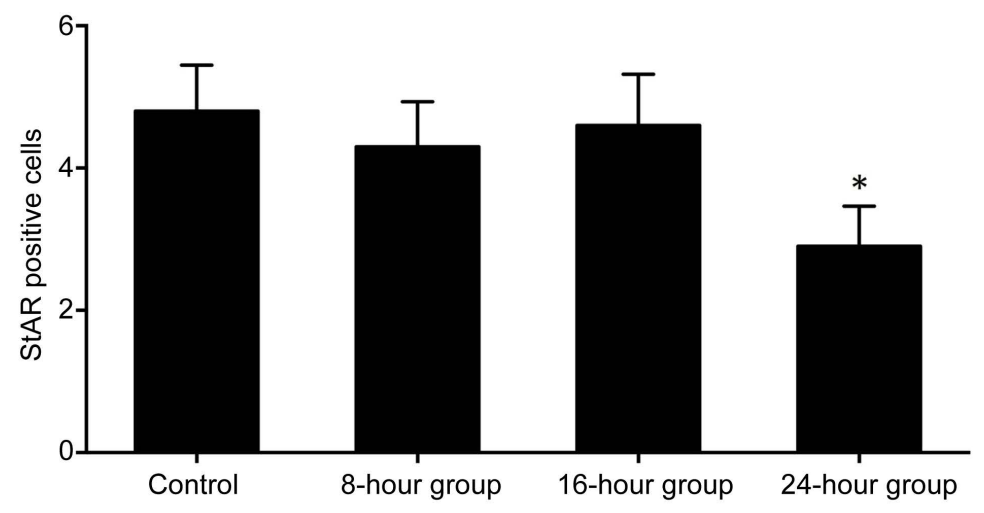

(b)

Figure 3. The StAR-positive Leydig cells in interstitial spaces. (a) The number of StAR-positive cells was determined. Scale bar, $100 \mu \mathrm{m}$; (b) The number of StAR-positive cellsper interstitial space. Results are shown asmean \pm standard error of the mean; ${ }^{\star} \mathrm{P}<$ 0.05 compared with control group; $n=10$ /group.

pression of one of the rate-limiting enzyme of testosterone synthesis, StAR.

Male infertility has been a public problem all over the world. Evaluating the possible side effects from use of some new technologies is critical to illuminatethis problem. With an increasing worldwide usage of cellphones, identifying the risks of cellphone is particularly crucial. Men always carry cellphones in their pants pockets which make them at a risk of exposure to harmful microwaves and might do harm to their fertility. Many researches have demonstrated that cellphone can affect the fertility of males. Ji-Geng Yan et al. has proved that carrying 


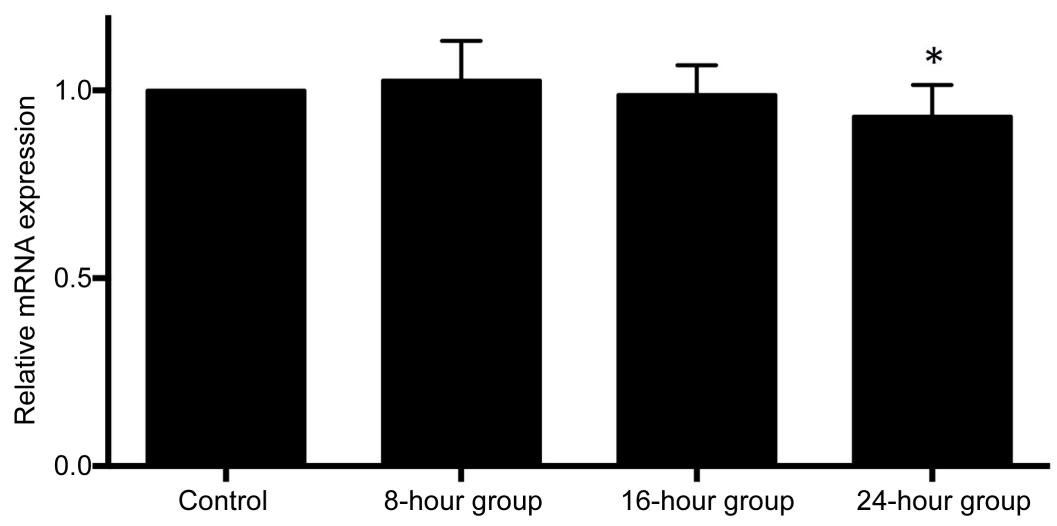

Figure 4. Quantitative real-time PCR analysis of StAR gene expression in testes of all groups. Data are expressed as mean \pm standard error of the mean; ${ }^{\star} \mathrm{P}<0.05$ compared with control group; $\mathrm{n}=10$ /group.

cellphones near reproductive organs could negatively affect fertility of male rats [11]. Challis has depicted that the electric field of CR can lead to a rapid heading and decrease sperm quality [12]. CR can also change the protein conformations and binding properties, and rise the production of reactive oxygen species (ROS) which might result in DNA damage [12] [13]. Dasdag, et al. has demonstrated that CR can induce histological changes in the testes of rats [14].

In the present study, we examined the sperm in the epididymis and observed that the sperm number and motility decreased significantly after long period exposure of CR (24-h group), but didn't change much after short or middle period exposure (8-h group and 16-h group). This result was similar with the outcomes of many other researches [15] [16]. As mentioned above, ITT level is roughly 50 - 100 times higher than serum testosterone level, and is very important for spermatogenesis. We continued to test ITT concentrations in each group after the experiment and found that ITT in 24-h group declined dramatically. No significant differences existed among other groups. The present results indicate that CR exposure can decrease testosterone synthesis, decline ITT concentration, suppress spermatogenesis, and then inhibit the quality of sperm.

In order to explore the mechanism of it, we set up to investigate the effects of CR exposure on the expression of the rate limiting enzyme, StAR, in the testis of adult male mice. To the best of our knowledge, this is the first time for this topic conducted on adult male animal mode. The results showed that no statistically changes of the StAR expression in testis tissue were seen in the 8-hour group and 16-hour group. Only the StAR expression in mice's testis in 24-hour group was statistically lower than that of the control group. These data showed that low and middle dosage exposure to CR did not affect the expression of StAR in the testis of adult male mice, but high dosage exposure to $\mathrm{CR}$ can reduce the expression of StAR in testis tissue, which indicated that CR might act in a dose-dependent manner.

Our study had several limitations. Further studies are needed to explore whether CR exposure affects rate-limiting enzymes of synthesis including cy- 
tochrome P450 cholesterol side-chain cleavage enzyme (P450scc) and $3 \beta$-hydroxysteroid dehydrogenase ( $3 \beta$-HSD) to make further efforts to elucidate the mechanism of it. Another limitation is that the number of mice in this study is relatively small, which may influence the outcomes of statistical analysis.

In conclusion, the present study demonstrated that high dose of CR exposure can suppress the express of StAR in the testis tissue, which lead to the decrease of the testosterone synthesis and the ITT concentration, and then inhibit spermatogenesis. This result indicates that reducing CR exposure time is very important to decrease the injury to male fertility.

\section{Acknowledgements}

This work was funded by the Fundamental Research Funds for the Central Universities (16ykpy44) and Natural Science Foundation of Guangdong Province (2016A030310142).

\section{References}

[1] Agarwal, A., Singh, A., Hamada, A. and Kesari, K. (2011) Cell Phones and Male Infertility: A Review of Recent Innovations in Technology and Consequences. International Brazilian Journal of Urology, 37, 432-454. https://doi.org/10.1590/S1677-55382011000400002

[2] Braune, S., Wrocklage, C., Raczek, J., Gailus, T. and Lücking, C.H. (1998) Resting Blood Pressure Increase during Exposure to a Radio-Frequency Electromagnetic Field. Lancet, 351, 1857-1858. https://doi.org/10.1016/S0140-6736(98)24025-6

[3] Oftedal, G., Wilén, J., Sandström, M. and Mild, K.H. (2000) Symptoms Experienced in Connection with Mobile Phone Use. Occupational Medicine, 50, 237-245. https://doi.org/10.1093/occmed/50.4.237

[4] Huber, R., Graf, T., Cote, K.A., et al. (2000) Exposure to Pulsed High-Frequency Electromagnetic Field during Waking Affects Human Sleep EEG. Neuroreport, 11, 3321-3325. https://doi.org/10.1097/00001756-200010200-00012

[5] Kesari, K.K. and Behari, J. (2010) Microwave Exposure Affecting Reproductive System in Male Rats. Applied Biochemistry and Biotechnology, 162, 416-428. https://doi.org/10.1007/s12010-009-8722-9

[6] Mailankot, M., Kunnath, A.P., Jayalekshmi, H., Koduru, B. and Valsalan, R. (2009) Radio Frequency Electromagnetic Radiation (RF-EMR) from GSM $(0.9 / 1.8 \mathrm{GHz})$ Mobile Phones Induces Oxidative Stress and Reduces Sperm Motility in Rats. Clinics (Sao Paulo), 64, 561-565. https://doi.org/10.1590/S1807-59322009000600011

[7] Zang, Z., Ji, S., Huang, S., Jiang, M. and Fang, Y. (2016) Impact of Cellphone Radiation on Sexual Behavior and Serum Concentration of Testosterone and LH in Male Mice. Occupational Diseases and Environmental Medicine, No. 4, 56-62. https://doi.org/10.4236/odem.2016.43007

[8] Kim, E.D., Crosnoe, L., Bar-Chama, N., Khera, M. and Lipshultz, L. (2013) The Treatment of Hypogonadism in Men of Reproductive Age. Fertility and Sterility, 99, 718-724. https://doi.org/10.1016/j.fertnstert.2012.10.052

[9] Trosić, I., Pavicić, I., Milković-Kraus, S., Mladinić, M. and Zeljezić, D. (2011) Effect of Electromagnetic Radiofrequency Radiation on the Rats' Brain, Liver and Kidney Cells Measured by Comet Assay. Collegium Antropologicum, 35, 1259-1264. 
[10] Bouji, M., Lecomte, A., Hode, Y., de Seze, R. and Villégier, A.S. (2012) Effects of 900 $\mathrm{MHz}$ Radiofrequency on Corticosterone, Emotional Memory and Neuroinflammation in Middle-Aged Rats. Experimental Gerontology, 47, 444-451. https://doi.org/10.1016/j.exger.2012.03.015

[11] Yan, J.G., Agresti, M., Bruce, T., Yan, Y.H., Granlund, A. and Matloub, H.S. (2007) Effects of Cellular Phone Emissions on Sperm Motility in Rats. Fertility and Sterility, 88, 957-964. https://doi.org/10.1016/j.fertnstert.2006.12.022

[12] Challis, L.J. (2005) Mechanisms for Interaction between RF Fields and Biological Tissue. Bioelectromagnetics, 7, S98-S106. https://doi.org/10.1002/bem.20119

[13] La Vignera, S., Condorelli, R.A., Vicari, E., D’Agata, R. and Calogero, A.E. (2012) Effects of the Exposure to Mobile Phones on Male Reproduction: A Review of the Literature. Journal of Andrology, 33, 350-356. https://doi.org/10.2164/jandrol.111.014373

[14] Dasdag, S., Ketani, M.A., Akdag, Z., et al. (1999) Whole-Body Microwave Exposure Emitted by Cellular Phones and Testicular Function of Rats. Urological Research, 27, 219-223. https://doi.org/10.1007/s002400050113

[15] Agarwal, A., Deepinder, F., Sharma, R.K., Ranga, G. and Li, J. (2008) Effect of Cell Phone Usage on Semen Analysis in Men Attending Infertility Clinic: An Observational Study. Fertility and Sterility, 89, 124-128. https://doi.org/10.1016/j.fertnstert.2007.01.166

[16] De Iuliis, G.N., Newey, R.J., King, B.V. and Aitken, R.J. (2009) Mobile Phone Radiation Induces Reactive Oxygen Species Production and DNA Damage in Human Spermatozoa in vitro. PLoS One, 4, e6446. https://doi.org/10.1371/journal.pone.0006446 